\title{
Chemical Composition and Structural Features of an Egyptian Funerary Mask from the Ptolemaic Period, Studied by SEM and EPMA
}

\author{
Faramarz S. Gard $^{1 *}$, Patricia B. Bozzano ${ }^{1,2}$, Silvia A. Dominguez ${ }^{1,2}$, Diego M. Santos ${ }^{3}$, María B. Daizo 3,4 \\ ${ }^{1}$ Comisión Nacional de Energía Atómica, Buenos Aires, Argentina \\ ${ }^{2}$ Institute Sabato-UNSAM \\ ${ }^{3}$ Universidad Pedagógica Nacional, Buenos Aires, Argentina \\ ${ }^{4}$ Universidad de Buenos Aires, Argentina \\ * Corresponding author: fsgard01@ gmail.com
}

In the current proceedings, we report complementary results of scanning electron microscopy (SEM) and electron probe micro analysis techniques (EPMA) on an Egyptian funerary mask from (3rd century BC), which was provided by the Museum of La Plata, see Fig. 1 (A).

SEM-energy dispersion spectroscopy (EDS) measurements, Back Scattering (BS), and Secondary Electron (SE) imaging were performed in a FEI Quanta 200 SEM with an EDAX Apollo detector, at low vacuum mode. Electron beam micro-analysis was carried out in a CAMECA SXFiveFE [1].

The combined results of these techniques provided an insight to the structural and metallurgical features of the golden leaf, which was used to resurfaced the mask. BS and SE images provided solid evidences of the ancient gold refinery practice, namely the cementation process. Fig. 1 (B) shows a BS image (magnification 1200X) obtained from the golden leaf. In addition to the micro-voids in the gold leaf, one can notice that it consists of at least two overlapping layers. The top layer has been ripped apart and rolled up at different locations.

Fig. 2 (A) shows a SE image (magnification 20000X) obtained from the golden leaf, where two typical micro-voids of dimension smaller than $5 \mu \mathrm{m}$ can be seen in more details. It can is also seen that the gold leaf consists of a mosaic of domains. Their origin can be related to the parting process of gold from silver in Electrum. Chemical composition of the sample was also measured, using SEM-EDS, see Fig. 2 (B), and EPMA-WDS techniques. In addition to Au and Ag (constitutional elements of the golden leaf), we have detected, $\mathrm{Al}, \mathrm{Fe}$, which can be attributed to the impurity in the plaster base of the mask, or to material residues, which have been used during the refinement process of gold. Small amount of $\mathrm{Mg}$, and relatively large quantity of $\mathrm{Ca}$ are believed to be related to the plaster in the form of magnesium calcite. Quartz $\left(\mathrm{Si}_{2} \mathrm{O}\right)$ impurity is manifested by detection of $\mathrm{Si}$ in the sample.

The relative concentration of gold to silver was measure to be 95\% to 5\%. EPMA-WDS confirmed the compositional elements of the sample measured by EDS, with better resolution, due to the principle of its measurement method. Furthermore, EPMA chemical mapping of gold and silver from the golden leaf provided a lateral distribution of those elements, which demonstrated an inhomogeneous characteristic of the Au-Ag alloy used to construct the mask.

References:

[1] FS Gard, et al., Surface and Interface Analysis, 52 (2019), p. 1. 


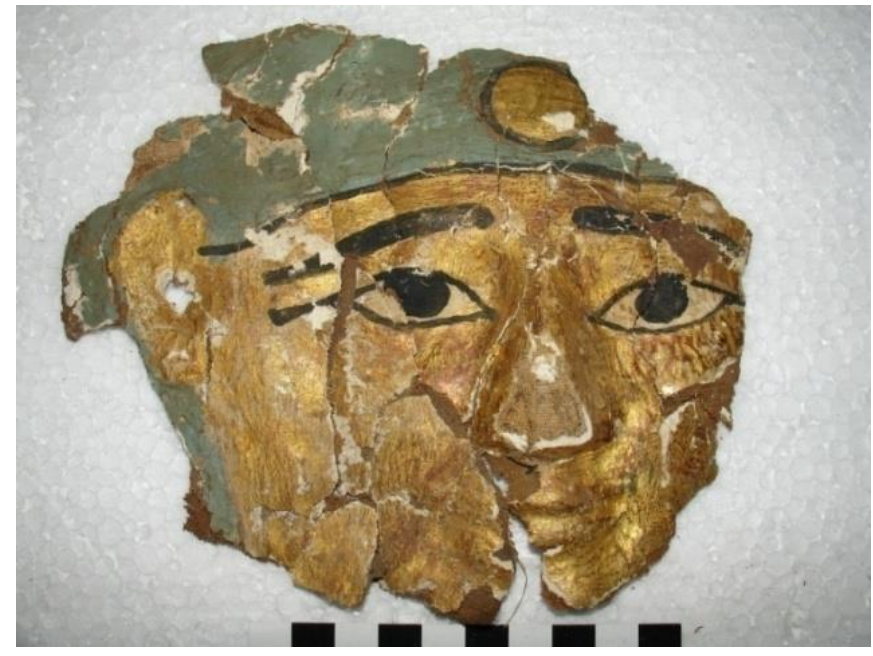

(A)

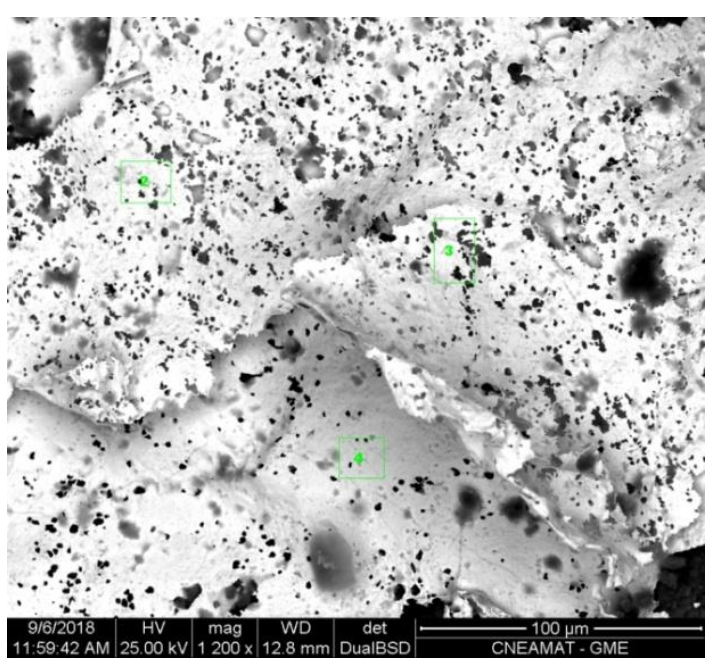

(B)

Figure 1: (A): Egyptian funerary mask preserved at Museum of La Plata. (B): Back scattering (BS) image (magnification $1200 \mathrm{M}$ ), from the golden part of the mask.

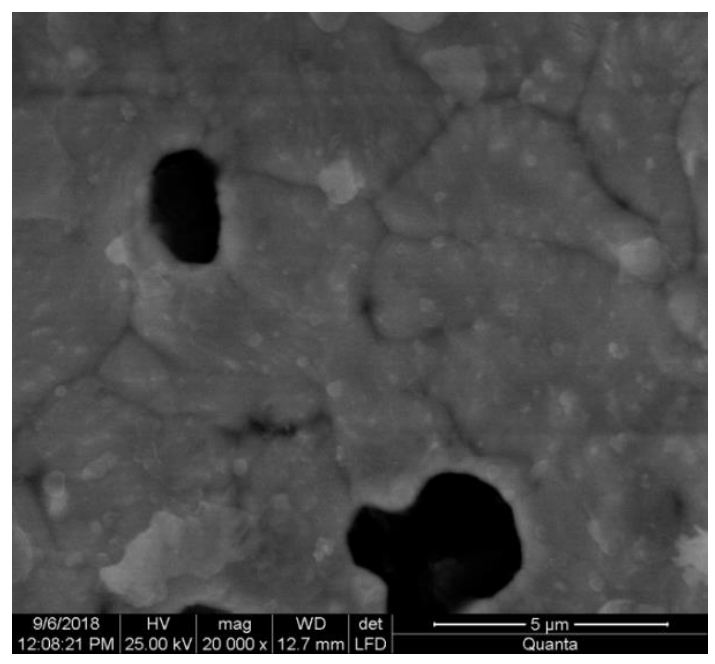

(A)

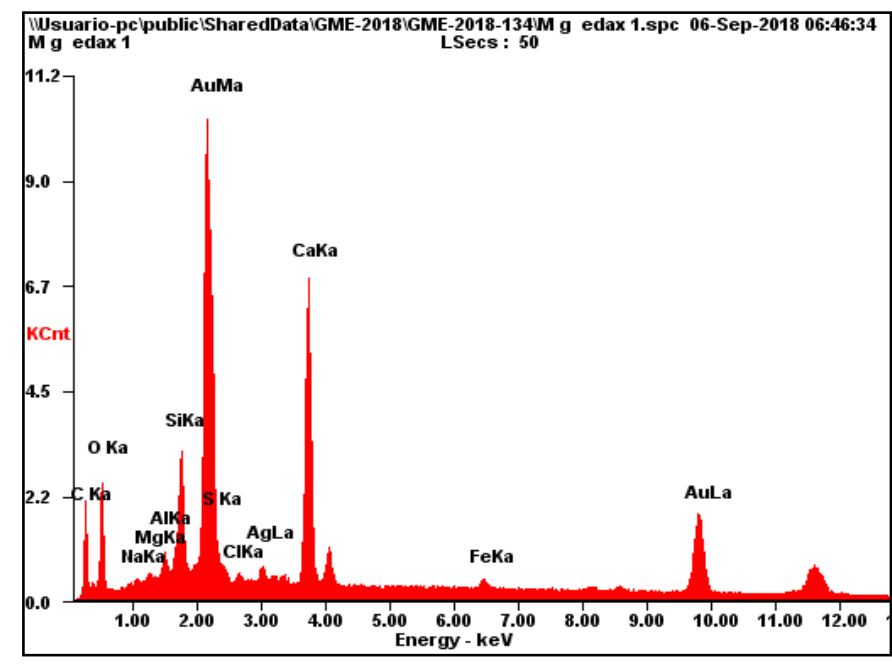

(B)

Figure 2: (A): Secondary electron (SE) image (magnification 20000X) obtained from the golden part of the mask, two small voids and domain boundaries can be seen in the image. (B): A characteristic SEMEDS spectrum acquired from the golden part of the mask sample. 